\title{
Eletroacupuntura na analgesia trans e pós-operatória de cadelas submetidas à ovariosalpingohisterectomia
}

\author{
[Electroacupuncture in trans and postoperative analgesia in bitches submitted \\ to ovariosalpingohisterectomy] \\ L.C.C. Santos ${ }^{1}$, L.S. Fernandes ${ }^{1}$, R.V. Sepulveda ${ }^{1}$, \\ V.G. Pereira ${ }^{1}$, R.B. Eleotério ${ }^{1}$, L.S.C. Favarato ${ }^{2}$ \\ ${ }^{1}$ Aluno de pós-graduação - Universidade Federal de Viçosa - Viçosa, MG \\ ${ }^{2}$ Universidade Federal de Viçosa - Viçosa, MG
}

\begin{abstract}
RESUMO
Objetivou-se com este estudo avaliar o efeito analgésico trans e pós-operatório da eletroacupuntura em onda denso-dispersa e frequências 3 e $200 \mathrm{~Hz}$, nos pontos E44, R3 e BP4, compará-lo com a analgesia promovida pelos pontos BP6, E36 e VB 34, e pela morfina, em cadelas submetidas à ovariosalpingohisterectomia (OSH). Teve-se como hipótese que a eletroacupuntura nos pontos E44, R3 e $\mathrm{BP} 4$ resultaria em controle da dor trans e pós-operatória melhor ou igual àquele promovido pela eletroacupuntura nos pontos BP6, E36 e VB34 e pela morfina. Trinta e seis cadelas submetidas à cirurgia eletiva de OSH foram distribuídas em blocos ao acaso em três grupos com 12 animais. Em dois grupos foi realizada eletroacupuntura denso-dispersa, com frequência de 3 e $200 \mathrm{~Hz}$, sendo que, no primeiro grupo (GEA), foram estimulados os pontos BP4, E44, R3 e, no segundo grupo (GEB), os pontos BP6, E36, VB34. Nesses dois grupos, foram administrados $1,5 \mathrm{~mL}$ de solução fisiológica por via IM. No terceiro grupo (GF), foi realizada acupuntura em três pontos falsos e o aparelho de eletroacupuntura foi acoplado, mas não foi ligado, e administrou-se morfina na dose de $0,5 \mathrm{mg} / \mathrm{kg}$, diluída em solução fisiológica de modo que o volume total foi de $1,5 \mathrm{~mL}$ por via IM. Foram avaliados os parâmetros cardiovasculares e respiratórios durante o período pré e transoperatório. A avaliação de dor pós-operatória foi realizada durante 12 horas, e a analgesia suplementar com morfina foi realizada quando o escore de dor alcançou valores iguais ou superiores a nove pontos (total de 27) de acordo com a "Escala de dor da Universidade de Melbourne" (EDUM). O grupo GEB apresentou pressão arterial estável, menor variação da Et $\mathrm{iso}_{\text {iso }} \mathrm{e}$ menor requerimento analgésico no pós-operatório. Nos grupos GEA e GF houve aumento da $\mathrm{Et}_{\text {iso }}$ e da pressão arterial nos momentos de maior manipulação cirúrgica. Os maiores escores de dor foram obtidos em MP0 (momento da extubação) no GEA (8) e em MP1(1h após a extubação) no GF (9). O GF apresentou o maior requerimento analgésico no pós-operatório e foram realizados 17 resgates em 6 animais. Os acupontos R3, E44 e BP4, utilizados na eletroacupuntura com a frequência de 3 e 200Hz, não são capazes de promover ação antinociceptiva satisfatória no período transoperatório. $\mathrm{O}$ efeito analgésico pós-operatório promovido pela eletroestimulação nos acupontos R3, E44 e BP4 é inferior ao proporcionado pelos acupontos E36, BP6 e VB34 e superior ao obtido com a morfina.
\end{abstract}

Palavras-chave: cão, acupontos, nocicepção, eletroestimulação, morfina

\section{ABSTRACT}

Thirty-six dogs undergoing elective ovariohysterectomy surgery were randomly distributed into 3 groups of 12 animals each. In the first group, dense-dispersed electroacupuncture was performed with a frequency of 3-200 Hz in SP4, ST44, KID3 points and 1.5mL of saline was given intramuscularly (GEA group). For the second group, dense-dispersed electroacupuncture was performed with a frequency of 3$200 \mathrm{~Hz}$ in SP6, ST36, GB34 points and $1.5 \mathrm{~mL}$ of saline given intramuscularly (GEB group). In the third group acupuncture was performed in 3 false points being the electroacupuncture device attached to the

Recebido em 14 de agosto de 2014

Aceito em 11 de junho de 2015

E-mail: lecalovi@gmail.com 
needles but not connected, and morphine was also administered at a dose of $0.5 \mathrm{mg} / \mathrm{kg}$ diluted in saline so that the total volume was $1.5 \mathrm{~mL}$ intramuscularly (GF group). Cardiovascular and respiratory parameters were evaluated during the pre and perioperative period. The evaluation of pain and postoperative sedation was performed for 12 hours and analgesic supplementation was performed whenever the pain score reached values above or equal to 9 points (in a total of 27) in accordance with the Scale of Pain of the University of Melbourne (EDUM). The GEB group had stable blood pressure and lower Et $t_{\text {iso }}$ value as well as less need for additional analgesia postoperatively. The GEA group showed the highest values of $E t_{\text {iso }}$ and showed the greatest need for additional analgesia in the immediate postoperative period (at the moment of extubation). The GF group required more supplementary analgesia postoperatively and showed higher sedation scores up to 4 hours after surgery. The KID3, ST44 and SP4 acupoints used in electroacupuncture with frequency 3-200 $\mathrm{Hz}$ are not able to promote satisfactory antinociceptive action in the perioperative period. Postoperative analgesic effect, promoted by electrical stimulation in acupoints KID3, ST44 and SP4, is inferior to that provided by acupoints ST36, SP6 and GB34 and superior to that obtained with morphine.

Keywords: dogs, acupoints, nociception, electrostimulation, morphine

\section{INTRODUÇÃO}

O controle da dor é amplamente discutido devido às considerações morais e éticas e aos efeitos deletérios produzidos pela dor, tais como estresse, alterações cardiovasculares, respiratórias e hidroeletrolíticas, já amplamente delineados na medicina humana e veterinária (Gaynor, 2000).

A dor pós-operatória é tradicionalmente controlada através da administração de medicamentos. Entre as opções de tratamento, os anti-inflamatórios e os opioides são as classes mais utilizadas; entretanto, têm uso limitado nos pacientes pediátricos, geriátricos, oncológicos e diabéticos, nos quais os efeitos indesejáveis desses fármacos, como sedação, depressão respiratória e alterações gastrointestinais tornamse mais preocupantes (Gaynor, 2000).

A busca por terapias coadjuvantes, como a analgesia profilática e terapia analgésica multimodal, incluindo a acupuntura, é de grande importância, por diminuir os efeitos adversos dos fármacos e evitar a hiperalgesia e alodinia (Pereira et al., 2008; Taffarel e Freitas, 2009; Sousa et al., 2012). Nesse sentido, a eletroacupuntura tem se mostrado uma terapia capaz de promover analgesia pós-operatória compatível com aquela obtida com a utilização de opioides, com a vantagem de ser um método prático, com mínimos efeitos indesejáveis e de custo acessível (Esper, 2005; Cassu et al., 2008; Pereira et al., 2008; Taffarel e Freitas, 2009).
$\mathrm{O}$ efeito analgésico produzido pela eletroacupuntura é influenciado pelo ponto estimulado (acupontos) e pela frequência de estimulação utilizada $(\mathrm{Hz} / \mathrm{s})$. Assim, o acuponto deve ser escolhido de acordo com o percurso do meridiano e a distribuição dos nervos que atravessam a área de ocorrência da dor (Taffarel e Freitas, 2009). Apesar dos crescentes estudos referentes à analgesia transoperatória pelo uso da eletroacupuntura, percebe-se a necessidade de estudos com a finalidade de avaliar diferentes acupontos, diferentes frequências de estimulação e a utilização destes em procedimentos cirúrgicos distintos.

Objetivou-se com este estudo avaliar o efeito analgésico trans e pós-operatório da eletroacupuntura em onda denso-dispersa e frequências 3 e $200 \mathrm{~Hz}$, nos pontos E44, R3 e BP4, compará-lo com a analgesia promovida pelos pontos BP6, E36 e VB 34, e pela morfina, em cadelas submetidas à OSH. Partiu-se da hipótese de que a eletroacupuntura nos pontos E44, R3 e BP4 resultaria em controle da dor trans e pós-operatória melhor ou igual àquele promovido pela eletroacupuntura nos pontos BP6, E36 e VB34 e pela morfina.

\section{MATERIAL E MÉTODOS}

Após aprovação da Comissão de Ética para uso de Animais da Instituição de Origem (protocolo $\mathrm{n}^{\mathrm{o}}$ 54/2013), foram selecionadas 36 cadelas dóceis, com indicação para OSH e peso corporal entre 6 e $22 \mathrm{~kg}$, mediante a normalidade dos exames físico e laboratorial (hemograma, função hepática e renal). 
Para a realização da cirurgia, os animais foram submetidos ao jejum alimentar de 12 horas e hídrico de oito horas. Antes do início da anestesia, os animais foram acomodados em baias individuais, em ambiente tranquilo, por pelo menos 30 minutos. Depois desse tempo, as características comportamentais dos animais foram observadas e foi avaliado o escore de dor basal (Momento basal - MB) por meio da "Escala de dor da Universidade de Melbourne" (EDUM) (Muir III, 2009).

$\mathrm{Na}$ sequência foi realizada a cateterização da veia cefálica para administração da solução de $\mathrm{NaCl}$ a $0,9 \%$, na velocidade de $6 \mathrm{~mL} \cdot \mathrm{kg}^{-1} \cdot \mathrm{h}^{-1}$, e a indução anestésica foi realizada com propofol (Propovan ${ }^{\circledR} \quad 10 \mathrm{mg} / \mathrm{mL}$, Cristália Produtos Químicos e Farmacêuticos Ltda., São Paulo, SP), $6 \mathrm{mg} . \mathrm{kg}^{-1}$, administrado por via intravenosa.

Após a intubação orotraqueal, a manutenção da anestesia foi realizada com isofluorano (Isoforine, Cristália Produtos Químicos e Farmacêuticos Ltda., São Paulo, SP) vaporizado em $100 \%$ de oxigênio, por meio de vaporizador calibrado (4.3, BD Hospitalar, Fila Friburgo, SP) e circuito valvular com reinalação total de gases. A concentração expirada de isofluorano $\left(\mathrm{ET}_{\text {iso }}\right)$ foi ajustada inicialmente em 1,2\% (Analisador de gases anestésicos, Lifewindow TM 600 vet, Digicare Animal Health, Flórida, USA) e os animais foram submetidos à ventilação controlada por pressão positiva intermitente (Aparelho de anestesia inalatória, Conquest, BD Hospitalar, Vila Friburgo, SP, Brasil), com pressão inspiratória de $15 \mathrm{cmH}_{2} \mathrm{O}$ e frequência respiratória adequada a manter a $\mathrm{EtCO}_{2}$ entre 35 e $45 \mathrm{mmHg}$.

A partir desse momento, os animais foram posicionados em decúbito dorsal sobre colchão térmico e foram distribuídos aleatoriamente em três grupos de doze animais cada. As cirurgias foram realizadas no período da manhã, em centro cirúrgico sem controle rigoroso de temperatura, na cidade de viçosa, cuja altitude é de $649 \mathrm{~m}$. No grupo GEA, foi realizada eletroacupuntura bilateral (EA) nos pontos E44, R3 e BP4 (agulhas de acupuntura 0,25 x $15 \mathrm{~mm}$, Arhon Din, São João Del Rei, MG), com frequência denso-disperso de 3 e $200 \mathrm{~Hz}$ (Eletroestimulador EL608, NKL Produtos Eletrônicos Ltda. Brusque, SC, Brasil) e aplicação de $0,05 \mathrm{~mL} \cdot \mathrm{kg}^{-1}$ de $\mathrm{NaCl} 0,9 \%$ por via intramuscular (IM). No grupo GEB, foi realizada eletroacupuntura bilateral nos pontos BP6, VB34 e E36, estimulados na mesma frequência utilizada no GEA e aplicação de $0,05 \mathrm{~mL} \cdot \mathrm{kg}^{-1} \mathrm{IM}$ de $\mathrm{NaCl}$ $0.9 \%$. No grupo GF, foi realizada a inserção bilateral das agulhas de acupuntura em três pontos falsos $(10$ a $20 \mathrm{~mm}$ lateral aos pontos de acupuntura utilizados no GEB) e o aparelho de eletroestimulação foi acoplado às agulhas, porém não foi ligado e administrou-se morfina $1 \%$ (Dimorf, Cristália Produtos Químicos e Farmacêuticos Ltda., São Paulo, SP) na dose de $0,5 \mathrm{mg} \cdot \mathrm{kg}^{-1} \mathrm{IM}$, diluída em solução fisiológica até um volume total de $1,5 \mathrm{~mL}$.

Após a administração intramuscular e início da eletroacupuntura, foi padronizado um tempo de espera de 20 minutos. Antes de iniciar o procedimento cirúrgico, foi realizado $\mathrm{o}$ pinçamento interdigital para avaliar a resposta ao estímulo nociceptivo, que foi considerada positiva quando houve aumento acima de $20 \%$ dos valores da pressão arterial e da frequência cardíaca. Em caso de resposta positiva, a concentração de isofluorano foi reajustada em $0,2 \%$ de $\mathrm{Et}_{\text {iso }}$ e aguardou-se um tempo de 5 minutos para nova avaliação. Quando a resposta foi negativa, a $\mathrm{Et}_{\mathrm{iso}}$ foi reduzida em $0,1 \%$ e teve início o procedimento cirúrgico. Esse tempo para o ajuste do anestésico inalatório foi computado. Após o início da cirurgia, a cada 5 minutos foram avaliados os mesmos parâmetros descritos e os mesmos critérios foram utilizados para o ajuste da $\mathrm{Et}_{\text {iso. }}$ A eletroacupuntura foi interrompida ao fim da cirurgia.

As variáveis fisiológicas (pressão parcial de dióxido de carbono ao final da expiração $\left(\mathrm{EtCO}_{2}\right)$, concentração expirada de isofluorano $\left(\mathrm{Et}_{\text {iso }}\right)$, frequência respiratória, frequência cardíaca, saturação da oxi-hemoglobina $\left(\mathrm{SpO}_{2}\right)$, pressão arterial sistólica, média e diastólica, e temperatura corporal) foram avaliadas nos momentos M1 a M9, conforme representado na Figura 1. Também foram computados a duração do procedimento cirúrgico e os tempos para extubação.

$\mathrm{O}$ efeito analgésico dos protocolos testados foi avaliado por 12 horas após a cirurgia (MP0 a MP12) com a utilização da "Escala de dor da Universidade de Melbourne" (EDUM), que avalia através de parâmetros fisiológicos e comportamentais e são atribuídos escores que 
podem somar de zero a 27 pontos, sendo zero o animal sem dor e 27, o máximo de dor. Os animais que receberam escores superiores a nove pontos foram tratados com morfina $1 \%$ na dose de $0,5 \mathrm{mg} \cdot \mathrm{kg}^{-1} \mathrm{IM}$ (analgesia suplementar), porém a dose máxima preconizada para cada animal foi de $1,5 \mathrm{mg} / \mathrm{kg}$, ou seja, no máximo três aplicações. $\mathrm{O}$ número de administrações adicionais de morfina e o tempo para a primeira administração após a cirurgia foram registrados.

Foi avaliado o grau de sedação nos mesmos momentos da avaliação da dor pós-operatória, utilizando-se sistema de escore descrito por Canôa (2009). Tal sistema descreve como "0" o animal não sedado, ou seja, representa o animal acordado, que se mantém em pé e caminha; o escore " 1 " representa aquele que se mantém em pé, mas incoordenado; o escore " 2 " está relacionado àquele animal que não consegue se manter em pé; o escore " 3 " é para o animal que consegue apenas manter a cabeça levantada; o escore "4" para o animal que, ao ser estimulado, somente abre os olhos; e o escore "5" é para o paciente mais sedado possível, que representa o animal sem resposta a estímulos.
As avaliações foram realizadas sempre pelo mesmo observador, com cada animal separadamente. O observador não teve conhecimento de qual grupo experimental o animal pertenceu. A análise estatística foi realizada inicialmente verificando se os dados atenderam às pressuposições Homogeneidade da Variância dos Erros e Normalidade dos Erros através dos testes de Cochran e Lilliford, respectivamente. Para os dados que atenderam a essas pressuposições, foi realizada uma Análise de Variância (ANOVA) e, havendo diferença significativa $(\mathrm{P}<0,05)$, foi aplicado o teste de Tukey. Esse procedimento foi realizado para a comparação entre os valores dos grupos experimentais em cada momento e comparação entre os momentos. Se os dados não atendessem às pressuposições, foi aplicado o teste $\mathrm{T}$ de Wilcoxon. Todos os testes foram realizados por meio do programa estatístico SAS 9.1 e foi utilizada significância de $5 \% \quad(\mathrm{p}<0,05)$. A confecção dos gráficos foi realizada utilizando o Microsoft Office Excel 2007.

\section{MOMENTOS EXPERIMENTAIS}

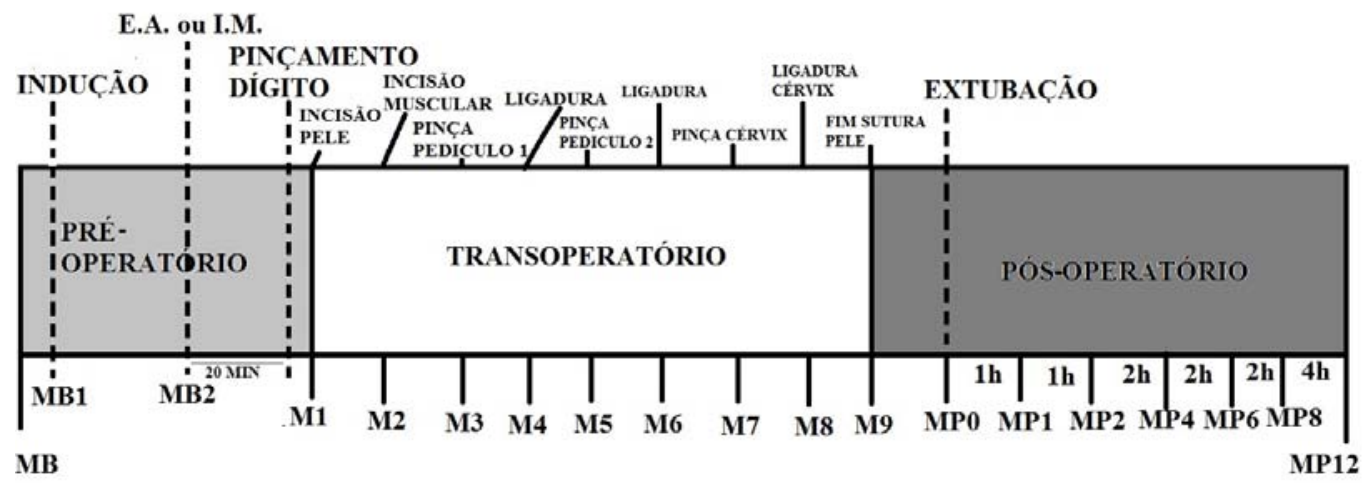

Figura 1. Representação esquemática dos momentos experimentais para a coleta de dados durante o estudo.

\section{RESULTADO E DISCUSSÃO}

A escolha dos acupontos E44, R3 e BP4 foi baseada na sua indicação para controle da dor abdominal e tratamento das doenças geniturinárias, na medicina tradicional chinesa (Chrisman e Xie, 2007), e por esse motivo optouse por utilizar tal associação com intuito de testar a sua eficiência analgésica.
No presente estudo, optou-se por utilizar a eletroestimulação denso-dispersa com frequência de 3 e $200 \mathrm{~Hz}$, pois, de acordo com Han (2004) e Cassu et al. (2012), essa combinação de duas frequências promovem a liberação de diferentes tipos de opioides endógenos relacionados ao controle da dor, resultando em um efeito terapêutico máximo. 
Um método indireto de avaliar a potência analgésica de um fármaco no período operatório é a determinação da redução do requerimento de anestésicos gerais inalatórios mensurada pela concentração final expirada ou a concentração alveolar mínima (CAM) (Thapun et al., 2014). Neste estudo, a $\mathrm{Et}_{\text {iso }}$ para o primeiro pinçamento interdigital foi padronizada em $1,2 \%$, valor este semelhante aos relatados para $\mathrm{CAM}_{\text {iso }} \mathrm{em}$ estudos com diversos fármacos e técnicas anestésicas, que variam de 1,15 a $1,8 \%$ (Credie et al., 2010; Monteiro et al., 2010; Campagnol, 2011).

$\mathrm{O}$ valor médio de $\mathrm{Et}_{\mathrm{iso}}$ que permitiu a incisão de pele foi de $2 \%$ (Fig. 2), valor este superior ao descrito por Muir III et al. (2003) e Aguado et al. (2011), sendo $1,38 \%$ e $0,7 \%$, respectivamente; entretanto, diferentemente dos estudos citados, o início da cirurgia só ocorreu após a ausência de variações cardiovasculares e respiratórias em resposta ao pinçamento interdigital, e não pela ausência de movimento voluntário a esse estímulo doloroso, o que justifica o valor mais alto da $\mathrm{Et}_{\text {iso }}$ no presente estudo.

O tempo gasto de MB2 a M1, para o ajuste do plano anestésico, o número de vezes que a cirurgia foi interrompida para reajuste da $\mathrm{Et}_{\mathrm{iso}}$, o tempo para extubação e a duração da cirurgia não apresentaram diferenças entre os grupos. Também não houve diferença significativa entre grupos nos valores da $\mathrm{Et}_{\text {iso }}$ utilizados para a manutenção anestésica $(2,4 \%$ em GEA e GF e $2,2 \%$ em GEB), o que demonstra que os protocolos utilizados proporcionaram ação antinociceptiva semelhante durante a cirurgia.

$\mathrm{Na}$ avaliação ao longo do tempo, o grupo GEB foi o único que apresentou valores estáveis em relação à $\mathrm{Et}_{\text {iso }}$ (Fig. 2), diferentemente dos grupos GEA e GF, nos quais foi necessário o aumento do requerimento do anestésico inalatório, a partir do pinçamento do primeiro pedículo ovariano. Como foi relatado anteriormente, os acupontos utilizados no GEB foram empregados em outros estudos (Lin et al., 2002; Cassu et al., 2008; Cassu et al., 2012), porém nestes não foi avaliada a $\mathrm{Et}_{\text {iso }} \mathrm{e}$, por esse motivo, não foi possível comparar os resultados encontrados com os dos demais autores. $\mathrm{O}$ acuponto E36, utilizado no GEB, é conhecido como acuponto multifuncional e por isso é utilizado no tratamento de diversas doenças e no controle da dor de qualquer origem (Saidah et al., 2013).

A pressão arterial e frequência cardíaca não apresentaram diferença entre os grupos. Entretanto, na avaliação ao longo do tempo, foi possível verificar que, nos grupos GEA e GF, a pressão arterial aumentou nos momentos de maior estímulo cirúrgico. Esse aumento pode ser atribuído à resposta neuroendócrina ao estímulo nociceptivo que leva a alterações cardiovasculares, como vasoconstrição e aumento da pressão arterial (Gaynor, 2000). O grupo GEB não apresentou variação na pressão arterial e esse resultado pode também indicar que o efeito antinociceptivo da eletroacupuntura nos pontos BP6, E36 e VB34 gerou um melhor controle da dor transoperatória, mantendo uma maior estabilidade cardiovascular quando comparado aos outros grupos. Não foi possível fazer uma correlação desses resultados com estudos relacionados, pois estes estudos avaliaram somente o controle da dor pósoperatória (Lin et al., 2002; Cassu et al., 2008; Cassu et al., 2012).

A frequência respiratória foi ajustada no ventilador mecânico, de forma a manter a $\mathrm{EtCO}_{2}$ entre 35 e $45 \mathrm{mmHg}$ e não houve diferença entre os grupos, ainda assim não é possível afirmar que não houve efeito dos protocolos testados sobre essa variável. Do mesmo modo, a saturação de oxi-hemoglobina não apresentou variações entre os grupos nem ao longo do tempo, mantendo valores acima de 95\%. Esse resultado provavelmente é consequência da manutenção dos animais em oxigênio a $100 \%$ e respiração controlada, favorecendo a oxigenação tecidual.

No pós-operatório, não foi observado aumento do escore de sedação; tal resultado pode ser atribuído ao fato de que, no presente estudo, não foram utilizados acupontos que objetivassem $o$ efeito sedativo, como o Yintang e o Vaso Governador 1 (VG1) (Luna et al., 2008); sendo assim, foi observada a redução dos escores de sedação ao longo do tempo, sem diferença entre os grupos.

Segundo Muir III (2003), o efeito sedativo dos opioides ocorre pela interação com receptores $\mu$ e $\kappa$, e segundo Monteiro et al. (2009), a dose utilizada, diferenças farmacocinéticas e o 
comportamento individual podem influenciar na sedação produzida por esses fármacos. Nesse sentido, seria esperado que o grupo GF apresentasse os maiores escores de sedação, visto que esse grupo foi o que recebeu o maior número de administrações de morfina, contudo esse fato não pode ser comprovado no presente estudo (Fig. 3).

A cirurgia de OSH é um procedimento que promove dor moderada e envolve estímulo somático e visceral; por esse motivo, a $\mathrm{OSH}$ torna-se adequada para os estudos clínicos de analgesia (Kotani et al., 2001). De acordo com White et al. (2004), a estimulação por eletroacupuntura é comprovadamente eficaz no alívio da dor somática e visceral em humanos e animais; resultado semelhante está relacionado à morfina, que foi eficiente no controle da dor pósoperatória (Dzikiti et al., 2006).

Na avaliação pós-operatória, os escores de dor e o requerimento analgésico permitem afirmar que o grupo GEB apresentou analgesia pósoperatória mais eficiente que os demais, pois um menor número de animais necessitou de analgesia adicional e dentre os animais tratados apenas um animal recebeu mais de uma administração de morfina (Fig. 4 e Fig. 5). Resultados semelhantes foram relatados por Cassu et al. (2012), que descreveram melhor controle da dor pós-operatória ao utilizar os mesmos acupontos testados nesse grupo, quando comparados a acupontos peri-incisionais.

Embora o menor efeito antinociceptivo no transoperatório tenha sido observado no GEA, no pós-operatório esse grupo apresentou menores escores de dor e consequentemente menor requerimento analgésico quando comparado ao GF. Esse resultado pode ser relacionado à liberação de opioides endógenos, inibição da dor na medula espinhal, tronco cerebral e outras áreas do sistema nervoso central todos promovidos pela eletroacupuntura (Cassu et al., 2012), como também à interferência da terapia analgésica adicional realizada em MP0 no GEA, que pode ter interferido nos escores de dor a partir de MP1 nesse grupo.

O GF apresentou o maior requerimento de analgésicos por animal (média de 2,8) e entre MP1 e MP8 foi o grupo que apresentou o maior escore de dor; e embora esse resultado não tenha obtido significado estatístico é importante considerá-lo. De acordo com Jones (2011), a morfina possui período hábil de 3 a 5 horas, o que pode ser associado ao maior escore de dor e consequentemente maior necessidade de analgésicos nesse grupo, visto que a administração preemptiva de morfina foi realizada pelo menos 2,3 horas antes de MP1. Tais resultados sugerem que a morfina na dose de $0,5 \mathrm{mg} / \mathrm{kg}$ por via intramuscular apresenta bom controle da dor por até 2 horas após sua aplicação.

Com relação à avaliação da dor pós-operatória, Pohl et al. (2011), utilizando a mesma escala, estabeleceram a pontuação de 13 para a realização de analgesia adicional e concluíram que uma pontuação menor (entre 6 e 7) deve ser considerada como indicativo de dor para a realização de analgesia adicional. Por outro lado, é importante ressaltar que efeitos adversos relacionados ao tratamento utilizado ou ao estresse, como sialorreia ou midríase, podem interferir na avaliação e pontuação final obtida, o que aumentaria os escores obtidos em animais que possivelmente não têm dor.

Mol et al. (2011) desconsideraram o diâmetro da pupila e a presença de salivação na EDUM por acreditarem que esses parâmetros poderiam ser influenciados por outros fatores além da dor. No presente estudo, o uso da morfina pode ter contribuído para a maior pontuação e consequentemente para o maior número de resgates analgésicos. Sialorreia e midríase são pontuadas na EDUM com 2 pontos cada; assim, neste estudo, tornou-se difícil diferenciar se esses parâmetros estavam relacionados à dor ou ao efeito colateral do opioide. O grau sedação pode ser um parâmetro utilizado para diferenciar o efeito do opioide e da dor, pois esses fármacos podem promover, além dos efeitos descritos, ação depressora central (Troncy et al., 2002). Porém, os escores de sedação obtidos no presente estudo não foram relevantes na determinação da influência do opioide nos escores de dor. Diante desse resultado, sugere-se que novos estudos sejam realizados modificando-se a avaliação, aumentando a pontuação referente à atividade do animal, presença de vocalização e a reação à palpação na escala de Melbourne, que parecem ser fatores mais fidedignos relacionados à dor, ou ainda excluindo os parâmetros diâmetro pupilar e salivação da avaliação. 


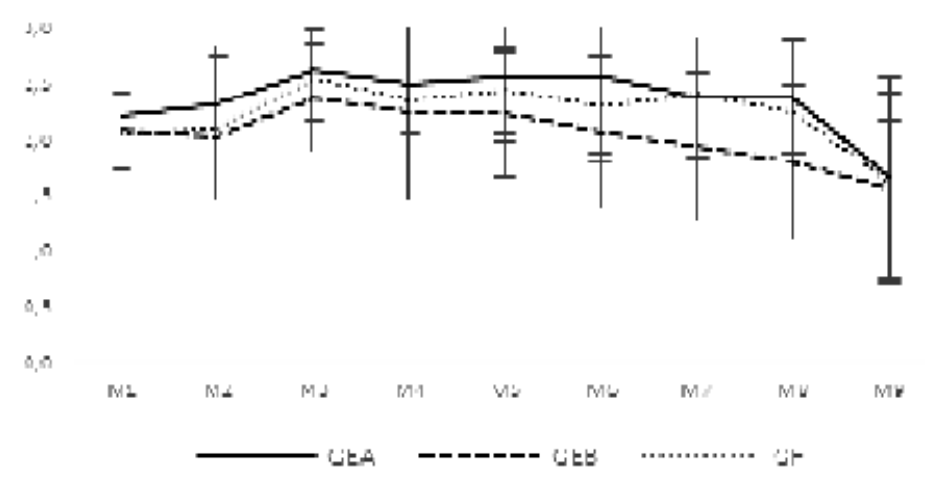

Figura 2. Representação da concentração expirada de isofluorano - $\mathrm{Et}_{\text {iso }}(\%)$ obtidos em cães anestesiados com propofol, isofluorano e tratados com eletroacupuntura nos pontos E44, R3, BP4 (GEA); BP6, VB34, E36 (GEB) ou em pontos falsos associados à morfina por via intramuscular (GF).

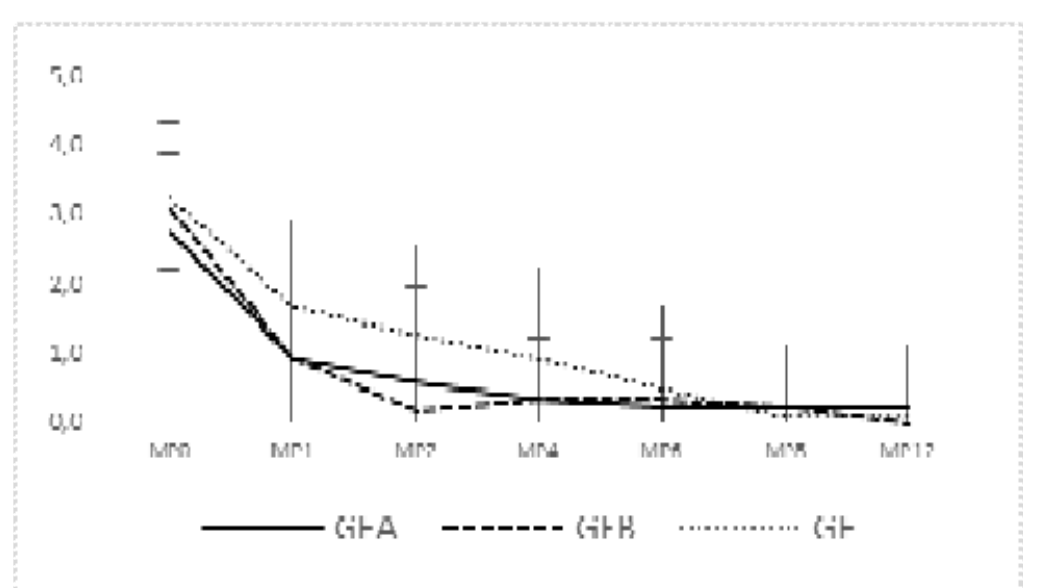

Figura 3. Representação dos escores de sedação apresentados por cadelas anestesiadas com isofluorano e tratadas com eletroacupuntura nos pontos E44, R3, BP4 (GEA); BP6, VB34, E36 (GEB) ou em pontos falsos associados à morfina por via intramuscular (GF).

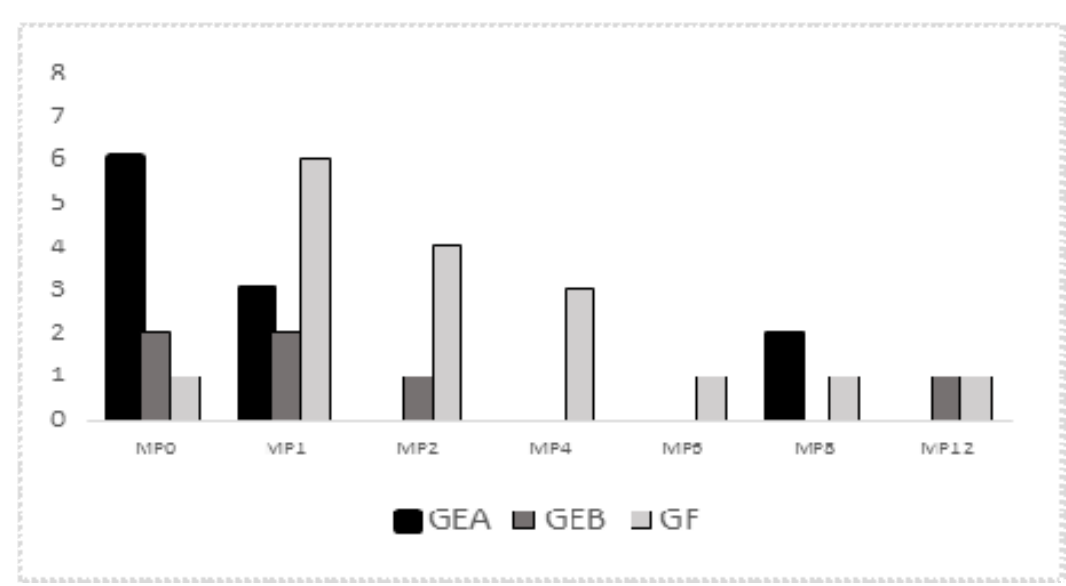

Figura 4. Representação do número de resgates apresentados por cadelas anestesiadas com isofluorano e tratadas com eletroacupuntura nos pontos E44, R3, BP4 (GEA); BP6, VB34, E36 (GEB) ou pontos falsos associados à morfina por via intramuscular (GF). 


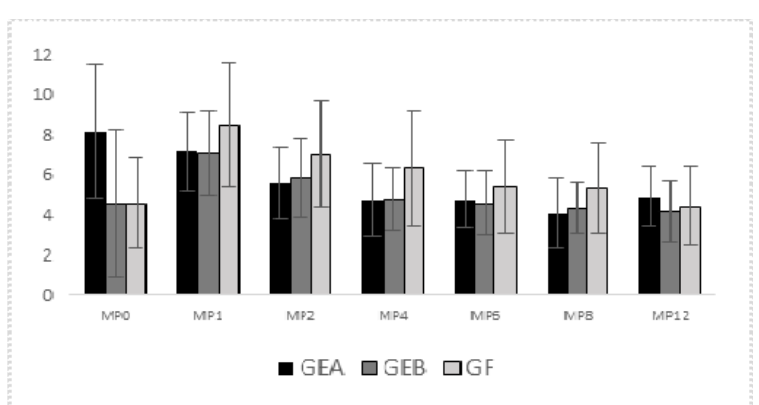

Figura 5. Representação dos escores de dor apresentados por cadelas anestesiadas com isofluorano e tratadas com eletroacupuntura nos pontos E44, R3, BP4 (GEA); BP6, VB34, E36 (GEB) ou pontos falsos associados à morfina por via intramuscular (GF).

\section{CONCLUSÃO}

Os acupontos E36, BP6 e VB34 utilizados na eletroacupuntura com frequência de 3 e $200 \mathrm{~Hz}$ apresentaram ação antinociceptiva satisfatória no período transoperatório de cadelas submetidas à OSH, assim como relatado em outros trabalhos. Os acupontos R3, E44 e BP4, utilizados na eletroacupuntura com a frequência de 3 a $200 \mathrm{~Hz}$, não foram capazes de promover ação antinociceptiva satisfatória. $\mathrm{O}$ efeito analgésico pós-operatório promovido pela eletroestimulação nos acupontos R3, E44 e BP4 foi superior ao obtido com a morfina $\mathrm{e}$ inferior ao proporcionado pelos acupontos E36, BP6 e VB34.

\section{AGRADECIMENTOS}

Os autores agradecem ao Conselho Nacional de Desenvolvimento Científico e Tecnológico (CNPq), à Coordenação de Aperfeiçoamento de Pessoal de Nível Superior (CAPES), à Fundação de Amparo à Pesquisa do estado de Minas Gerais (FAPEMIG) pelo apoio financeiro.

\section{REFERÊNCIAS}

AGUADO, D.; BENITO, J.; SEGURA, I. A. S.; Reduction of the minimum alveolar concentration of isoflurane in dogs using a constant rate of infusion of lidocaine-ketamine in combination with either morphine or fentanyl. The Veterinary Journal, v.189; p.63-66, 2011.

CAMPAGNOL, D. Farmacologia clínica da metadona peridural e intravenosa em cães. 2011. 182f. Tese (Doutorado em Anestesiologia Veterinária) - Universidade Estadual de São Paulo, Botucatu, SP.
CANÔA, J.T.B. Analgesia peridural com ropivacaína associada ao midazolam em cadelas submetidas a ovário-salpingo-histerectomia. 2009. 90f. Dissertação (Mestrado em Ciência Animal) - Universidade Estadual Paulista, Araçatuba, SP.

CASSU, R.N.; LUNA, S.P.L.; CLARK, R.M.O.; KRONKA, S.N. Electroacupuncture analgesia in dogs: is there a difference between uni- and bilateral stimulation? Vet. Anaesth. Analg. v.25, p.52-61, 2008 .

CASSU, R.N.; SILVA, D.A., GENARI FILHO, T.; STEVANIN, H. Electroanalgesia for the postoperative control pain in dogs. Acta Cir. Bras., v.27, p.43-48, 2012.

CHRISMAN, C.; XIE, H. Canine transpositional acupoints. In: XIE, H.; PREAST, V. Xie's veterinary acupuncture. Oxford: Blackwell, 2007. p.129-190, 2007.

CREDIE, R.G.; TEIXEIRA NETO, F.J. FERREIRA, T.H. et al. Effects of methadone on the minimum alveolar concentration of isoflurane in dogs. Vet. Anaesth. Analg., v.37, p.240-249, 2010.

DZIKITI, T.B.; JOUBERT, K.E.; VENTER, L.J.; DZIKITI, L.N. Comparison of morphine and carprofen administered alone or in combination for analgesia in dogs undergoing ovariohysterectomy. J. S. Afr. Vet. Assoc., v.77, p.120-126, 2006.

ESPER, G.V.Z. Estudo comparativo do emprego da eletroacupuntura e cloridrato de meperidina na analgesia de cadelas submetidas a castração cirúrgica. 2005. 85f. Dissertação - Curso de PósGraduação em Ciências Veterinárias, Universidade Federal do Paraná, Curitiba, PR. 
GAYNOR, J. S. Acupuncture for management of pain. Manag. Pain, v.30, p.875-884, 2000.

HAN, J.S. Acupuncture and endorphins. Neurosci. Lett., v.361, p.258-261, 2004.

JONES, R.S.; Epidural analgesia in dog and cat. Vet. J., v.161, p.123-131, 2001.

KOTANI, N.; HASHIMOTO, H.; SATO, Y. et al. Preoperative intradermal acupuncture reduces postoperative pain, nausea and vomiting, analgesic requirement, and sympathoadrenal responses. Anestesiology, v.95, p.349-356, 2001.

LIN, J.G.; LO, M.W.; WEN, Y. R. et al. The effect of high and low frequency electroacupunture in pain after lower abdominal surgery. Pain. v.99, p.509-514, 2002.

LUNA, S.P.L.; ANGLI, A.L.; FERREIRA, C.L. et al. Comparison of pharmacopuncture, aquapuncture and acepromazine for sedation of horses. Evid. Based Complement. Alternat. Med., v.5, p.267-272, 2008.

MOL, X.; FRESNO, L.; GARCÍA, F. et al. Comparison of subcutaneous and transdermal administration of buprenorphine for pre-emptive analgesia in dogs undergoing elective ovariohysterectomy. Vet. J., v187, p.124-128, 2011.

MONTEIRO, E.R.; RODRIGUES JUNIOR, A.; ASSIS, H.M.Q. et al. Comparative study on the sedative effects of morphine, methadone, butorphanol or tramadol, in combination with acepromazine, in dogs. Vet. Anaesth. Analg., v.36, p.25-33, 2009.

MONTEIRO, E.R.; TEIXEIRA-NETO, F.J., CAMPAGNOL, D. et al. Effects of remifentanil on the minimum alveolar concentration of isoflurane in dogs. Am. J. Vet. Res., v71, p.150-6, 2010.

MUIR III, W.W. Drogas utilizadas para tratar a dor. In: GAYNOR, J.S.; MUIR III, W.W. Manual de controle da dor em medicina veterinária. 2.ed. São Paulo: Medvet, 2009. p.141-162.

MUIR III, W.W.; WIESE, A.J.; MARCH, P.A. Effects of morphine, lidocaine, ketamine, and morphine-lidocaine-ketamine drug combination on minimum alveolar concentration in dogs anesthetized with isoflurane. Am. J. Vet. Res., v.64, p.1155-1160, 2003.
PEREIRA, K.S.; CARNEIRO, N.M.; GIL, J.N. Estudo do uso da acupuntura como analgesia preemptiva para pacientes de cirurgia de terceiros molares inferiores com osteotomia. Rev. Dor, v.9, p.1283-1298, 2008.

POHL, V.H.; CARREGARO, A.B.; LOPES, C. et al. Correlação entre as escalas visual analógica, de Melbourne e filamentos de Von Frey na avaliação da dor pós-operatória em cadelas submetidas à ovariossalpingohisterectomia. Ciênc. Rural, v.41, p.154-159, 2011.

SAIDAH, R.; CHUEIRE, A.G.; REJAILI, W.A. et al. Acupuntura em relação a dor, atividade física e a necessidade de apoio para a marcha no pós-operatório das cirurgias artroscópicas no joelho. Acta Ortop. Bras., v.11, p.5-10, 2013.

SOUSA, N.R.; LUNA, S.P.L.; CÁPUA, M.L.B. et al. Analgesia da farmacopuntura com meloxicam ou da aquapuntura preemptivas em gatas submetidas à ovariosalpingohisterectomia. Ciênc. Rural, v.42, p.1231-1236, 2012.

TAFFAREL, M.O.; FREITAS, P.M.C. Acupuntura e analgesia: aplicações clínicas e principais acupontos. Ciênc. Rural, v.39, p.26652672, 2009.

THAPUN, P.; DURONGPHONGTORN, S.; KALPRAVIDH, M. The effects of transdermal fentanyl patches on required isoflurane concentration when compared with intramuscular morphine during patelar luxation repair in dogs. Thai. J. Vet. Med., v.44, p.85-93, 2014.

TRONCY, E.; JUNOT, S.; KEROACK, S.; SAMMUT, V.; PIBAROT, P. GENEVOIS, J.P; CUVELLIEZ, S. Results of preemptive epidural administration of morphine with or without bupivacaine in dogs and cats undergoing surgery: 265 cases (1997- 1999). J. Am. Vet. Med. Assoc., v.221, p.666-672, 2002.

WHITE $P$, LEWITH G, PRESCOTT $P$, CONWAY J. Acupuncture versus placebo for the treatment of chronic mechanical neck pain: a randomized, controlled trial. Ann. Intern. Med.; v.141, p.911-9, 2004. 